\title{
Acquisition of tolerance to egg and peanut in African food-allergic children with atopic dermatitis
}

\author{
C L Gray, ${ }^{1} \mathrm{MB} \mathrm{ChB}, \mathrm{FRCPCH}, \mathrm{MSc}, \mathrm{PhD} ; \mathrm{M}$ E Levin, ${ }^{1} \mathrm{MB} \mathrm{ChB}$, FCPaed (SA), PhD; G du Toit, ${ }^{2} \mathrm{MB} \mathrm{ChB}, \mathrm{FRCPCH}$ \\ ${ }^{1}$ Division of Allergology, Department of Paediatrics and Child Health, Red Cross War Memorial Children's Hospital, Faculty of Health Sciences, \\ University of Cape Town, South Africa \\ ${ }^{2}$ Department of Paediatrics and Child Health, Red Cross War Memorial Children's Hospital, Faculty of Health Sciences, \\ University of Cape Town, South Africa; and King's College London, King's Health Partners, MRM and Asthma UK Centre in Allergic \\ Mechanisms of Asthma, and Department of Paediatric Allergy, Guy's and St Thomas' NHS Foundation Trust, London, UK
}

Corresponding author: C L Gray (claudiagray.paediatrics@gmail.com)

\begin{abstract}
Background. There are no previous data on tolerance development in children with atopic dermatitis (AD) and concomitant food allergy in low- and middle-income settings.

Objectives. To determine the rate of tolerance acquisition to egg and peanut 5 years after diagnosing food allergies in South African (SA) children with $\mathrm{AD}$, and to explore factors influencing tolerance acquisition.

Methods. Five years after first diagnosing food allergy in 37 SA children with egg and/or peanut allergy, they were reassessed for their allergies by questionnaire, skin-prick tests (SPTs) and ImmunoCAP-specific IgE (sIgE) tests (Thermo Fisher Scientific/Phadia, Sweden) to egg white, ovomucoid, peanut and Arachis hypogaea allergen 2 (Ara h 2), and incremental food challenges.

Results. Eighteen of 25 originally egg-allergic patients and 19 of 24 originally peanut-allergic children were followed up at a median age of 8 years and 3 months and 9 years and 6 months, respectively. A high percentage of children (72.2\%) outgrew their egg allergy, and $15.8 \%$ outgrew their peanut allergy. Allergic comorbidity remained high, with asthma increasing over time, and $\mathrm{AD}$ remaining moderate in severity in the cohort overall. At diagnosis, sIgE egg white $\leq 9.0 \mathrm{kU} / \mathrm{L}$ and $\operatorname{sgE}$ ovomucoid $\leq 2.0 \mathrm{kU} / \mathrm{L}$ were associated with tolerance development to egg 5 years later. At follow-up, sIgE egg white $\leq 0.70 \mathrm{kU} / \mathrm{L}$, sIgE ovomucoid $\leq 0.16 \mathrm{kU} / \mathrm{L}$, SPT egg-white extract $\leq 1 \mathrm{~mm}$ and SPT fresh egg $\leq 5 \mathrm{~mm}$ were associated with tolerance. At diagnosis, sIgE Ara h $2 \leq 1.7 \mathrm{kU} / \mathrm{L}$ and SPT peanut $\leq 10 \mathrm{~mm}$ were associated with tolerance development to peanut 5 years later. At follow-up, sIgE peanut $\leq 0.22 \mathrm{kU} / \mathrm{L}$, sIgE Ara h $2 \leq 0.18 \mathrm{kU} / \mathrm{L}$ and SPT peanut $\leq 5.5 \mathrm{~mm}$ were associated with tolerance.

Conclusions. Egg allergy was outgrown in $72.2 \%$ and peanut allergy in $15.8 \%$ of SA children 5 years after diagnosis of AD. This is in keeping with findings derived from studies in higher socioeconomic settings, and can help to guide the counselling of patients with allergies to these foods of high nutritional value.
\end{abstract}

S Afr Med J 2019;109(5):323-327. DOI:10.7196/SAMJ.2019.v109i5.13339

Egg and peanut allergies are among the most common childhood food allergies. The estimated prevalence of egg allergy is $0.5-8.9 \%$ in early childhood, ${ }^{[1-6]}$ while peanut allergy prevalence has a wide range according to geographical area, generally $\sim 0.5-2.0 \%$, but as high as $5.9 \%$ in some countries such as Sweden. ${ }^{[7-9]}$ Figures are higher, $\sim 15$ $30 \%$ for egg allergy and $10-25 \%$ for peanut allergy, in children with moderate to severe atopic dermatitis (AD). ${ }^{[10-12]}$

The natural history of egg and peanut allergy varies significantly. Egg allergy has a good overall prognosis, with half of children becoming tolerant by 3 years, $66 \%$ by 5 years ${ }^{[13]}$ and $\sim 80 \%$ by adolescence. The prognosis may be more guarded in those attending a specialised allergy clinic. A retrospective chart review of 881 children with egg allergy in a North American tertiary referral centre showed a slower development of tolerance $-4 \%$ by 4 years, $12 \%$ by 6 years, $37 \%$ by 10 years and $68 \%$ by 16 years. ${ }^{[14]}$ In a tertiary centre in Japan, $60 \%$ of children with hen's egg allergy at age 6 years were found to outgrow the allergy by age 12 years; the prognosis was more guarded in those with a past history of an anaphylactic reaction, in whom $44 \%$ outgrew the allergy by the age of 12 years. ${ }^{[15]}$

Clinical and laboratory factors that affect the development of tolerance to egg include milder symptoms on egg ingestion, skin test and egg IgE size at diagnosis, rate of change of IgE levels and presence of multiple food allergies. ${ }^{[16-18]} \mathrm{Gal} \mathrm{d} 1$ (ovomucoid) is the dominant allergen in egg white. Studies addressing persistence of egg allergy found higher concentrations of IgE anti-ovomucoid antibodies in persons with persistent allergy than in those who outgrew their egg allergy. ${ }^{[19]}$

Peanut allergy is persistent in the majority of cases, and only outgrown in $\sim 20 \%$ of patients. ${ }^{[20-23]}$ A low peanut-specific IgE (sIgE) at initial diagnosis may predict tolerance acquisition. A North American study of 84 patients with peanut allergy showed that those with a peanut $\operatorname{IgE}$ level $<5 \mathrm{kU} / \mathrm{L}$ at diagnosis had a $50 \%$ chance of outgrowing their allergy. ${ }^{[2]}$ Those who had an anaphylactic reaction to peanut were less likely to outgrow their allergy. ${ }^{[22]}$

A further North American study that followed up 223 patients with peanut allergy showed that $21.5 \%$ outgrew their allergy. ${ }^{[25]}$ Peanut sIgE was not different at diagnosis, but significantly lower in the tolerance group at the time of rechallenge (median $0.69 \mathrm{kU} / \mathrm{L}$ in those who developed tolerance v. $2.06 \mathrm{kU} / \mathrm{L}$ in those with persistent allergy).

Because of the persistent nature of peanut allergy, education regarding peanut avoidance and treatment of adverse reactions is necessary in all cases of clinical peanut hypersensitivity. Nonetheless, the rate of accidental ingestion is high. In a follow-up study of 83 children with peanut allergy, $58 \%$ had experienced adverse reactions from 
accidental peanut exposure after 5 years, and half of those had experienced potentially life-threatening reactions, regardless of the nature of their initial reaction. ${ }^{[22]}$

Data on food allergy in low- and middle-income countries are sparse. In a South African (SA) study of food allergy prevalence in children with moderate to severe AD, $25 \%$ had egg allergy and $24 \%$ peanut allergy. ${ }^{[12]}$ In this cohort, commonly used positive predictive cut-off values in skin-prick tests (SPTs) to egg white and peanut were poorly predictive for true allergy, leading the authors to propose that higher cut-off values should be used in such settings.

There are no data on factors influencing tolerance development in children with AD and concomitant food allergy in low- and middleincome settings. Many common food allergens are nutritious and affordable, and alternatives are expensive and not always readily available; hence, knowledge of the natural history of common food allergies is of particular importance in this setting.

This study aimed to determine the rate of acquisition of egg and peanut allergy tolerance in SA children with $\mathrm{AD}$ and concomitant food allergy. A secondary objective was to explore factors, at diagnosis and follow-up, which affect tolerance acquisition.

\section{Methods}

Children (6 months - 10 years of age) with moderate (SCORing Atopic Dermatitis (SCORAD) 15 - 40) to severe (SCORAD >40) $\mathrm{AD}$ and concommitant food allergy were followed up 5 years after diagnosis. Participants had previously been assessed for food allergies by detailed history, SPTs and ImmunoCAP tests (Thermo Fisher Scientific/Phadia, Sweden) for allergen sIgE and incremental open food challenges. ${ }^{[12,26,27]}$ Children with a prior diagnosis of egg and/or peanut allergy were reassessed by detailed questionnaire, SPTs and ImmunoCAP tests. SPTs were performed to egg white extract and peanut extract (ALK Abelló, Spain), as well as fresh raw egg white. SPT results were recorded as mean wheal diameter (largest diameter and perpendicular) minus mean wheal diameter of the negative control. All participants underwent ImmunoCAP tests to egg white, ovomucoid, peanut and Arachis hypogaea allergen 2 (Ara h 2). All patients also underwent an incremental open food challenge to egg and/or peanut, depending on their original allergy.

For egg, the challenge food was given in the form of scrambled egg, starting with a lip challenge, followed by incremental increases every 15 - 20 minutes, from $0.5 \mathrm{~g}$ to $30 \mathrm{~g}$ scrambled egg ( $50 \mathrm{mg}-3 \mathrm{~g}$ eggwhite protein), over 2 hours.

For peanut, the challenge food was given in the form of peanut butter, starting with a lip challenge, followed by increases from $0.1 \mathrm{~g}$ to $17 \mathrm{~g}$ of peanut butter ( $25 \mathrm{mg}-4 \mathrm{~g}$ peanut protein) over 2 hours, with dose increments every 15 - 20 minutes.

\section{Ethical approval}

This study was approved by the University of Cape Town's Human Research Ethics Committee (ref. no. 224/2015).

\section{Statistical analysis}

Data distribution was asymmetrical. The $\chi^{2}$ test and the Wilcoxon rank sum test were used to determine statistical significance $(p<0.05)$. Medians and interquartile ranges were used for descriptive statistics.

\section{Results}

\section{Egg allergy}

Follow-up rates and acquisition of tolerance

Eighteen of 25 participants previously diagnosed with egg allergy completed a full follow-up, including food challenges $(72.0 \%$ follow- up rate). The other 7 were lost to follow-up. There were no significant differences in baseline characteristics between those followed up and those lost to follow-up, including original eczema severity by SCORAD, age at study entry, asthma prevalence, SPT size to egg and raw egg, sIgE to egg white and ovomucoid at diagnosis, and severity of previous reactions (Table $\mathrm{E}^{\star}$ ).

The median age of those followed up and undergoing challenge was 8 years and 3 months (99 (interquartile range (IQR) 82 - 116) months).

None of the patients followed up had experienced a severe reaction to egg (anaphylaxis) at the time of study entry. Nine of 18 patients $(50.0 \%)$ had accidentally ingested whole egg during the 5 years since the initial study, of whom 5 had reacted at the time of accidental ingestion, none with an anaphylactic reaction.

Overall, 13/18 participants (72.2\%) passed their egg challenge and were classified as having outgrown their egg allergy. Nine of 10 (90.0\%) of those of mixed ethnicity outgrew their allergies at a median age of 82 (IQR 76 - 118) months and 4/8 (50.0\%) of the black Africans outgrew their egg allergy at a median age of 105 (IQR 99 - 113) months (inter-ethnic difference in outgrowing egg allergy, $p=0.06$ ).

\section{Allergic comorbidity}

Eleven of 18 participants (61.0\%) had asthma at reassessment compared with $7 / 18$ (38.9\%) with asthma at the time of the original study; hence, there was a $22.0 \%$ increase in asthma over 5 years in this group. At the time of diagnosis, 3/13 (23.1\%) of those who later outgrew their egg allergy had asthma, and $4 / 5$ (80.0\%) of those with persistent egg allergy had asthma $(p=0.027)$. At follow-up, the difference in asthma rates was no longer statistically significant: of those who outgrew their egg allergy, 7/13 had asthma (53.8\%), and of those who did not outgrow their egg allergy, $4 / 5$ had asthma ( $80.0 \% ; p=0.33)$.

The median SCORAD at study entry in the group who outgrew their egg allergy was 48 (IQR 44 - 52) compared with 35 (IQR 34 - 48) in those who did not outgrow their egg allergy $(p=0.255)$. At followup, the median SCORAD in those who outgrew their egg allergy (26; IQR 15 - 30) and those who did not (30; IQR 29 - 44) had decreased over the course of the 5 years.

\section{Specific IgE tests to egg white and ovomucoid at diagnosis and} follow-up in relation to tolerance acquisition

The sIgE to egg white and ovomucoid at diagnosis and follow-up is compared in those who outgrew their egg allergy and those with persistent allergy (Table 1).

\section{Skin-prick tests to egg-white extract and fresh egg at diagnosis and follow-up in relation to tolerance acquisition} SPTs to egg-white extract and fresh raw egg white at diagnosis and follow-up are compared in those who outgrew their allergy with those with persistent allergy (Table 1).

\section{Ratios of follow-up results to original results}

The ratios of median sIgE egg at follow-up and diagnosis are depicted in Table 1; similar ratios for ovomucoid sIgE, SPT to egg-white extract and SPT to fresh raw egg white are depicted in Table 1.

\section{Peanut allergy \\ Follow-up rates and acquisition of tolerance}

Nineteen of 24 participants previously diagnosed with peanut and egg allergy completed a full follow-up, including a peanut butter food challenge $(79.2 \%$ follow-up rate). There were no significant differences in baseline characteristics between those followed up and those lost to follow-up with regard to age at study entry, asthma 
Table 1. Factors affecting tolerance acquisition in egg allergy

\begin{tabular}{|c|c|c|c|}
\hline Factor & $\begin{array}{l}\text { Children who } \\
\text { acquired tolerance } \\
\text { to egg }\end{array}$ & $\begin{array}{l}\text { Children with } \\
\text { persistent egg allergy }\end{array}$ & Difference ( $p$-value) \\
\hline Asthma at diagnosis, $\%$ & 23.1 & 80.0 & 0.027 \\
\hline Asthma at follow-up, \% & 53.8 & 80.0 & 0.33 \\
\hline Median SCORAD at diagnosis (IQR) & $48(44-52)$ & $35(34-48)$ & 0.255 \\
\hline Median SCORAD at follow-up (IQR) & $26(15-30)$ & $30(29-44)$ & 0.17 \\
\hline sIgE egg white at diagnosis, kU/L (IQR) & $9.2(6.2-40.0)$ & $100(100-100)^{*}$ & 0.002 \\
\hline sIgE egg white at follow-up, kU/L (IQR) & $0.7(0.3-2.0)$ & $20.7(8.6-49.9)$ & 0.007 \\
\hline sIgE ovomucoid at diagnosis, kU/L (IQR) & $2.0(0.3-6.3)$ & $100.0(63.2-100.0)^{*}$ & 0.001 \\
\hline sIgE ovomucoid at follow-up, kU/L (IQR) & $0.2(0.1-0.4)$ & $23.3(4.3-66.4)$ & 0.006 \\
\hline SPT egg-white extract at diagnosis, mm (IQR) & $6(5-6)$ & $6(1-7)$ & 0.840 \\
\hline SPT egg-white extract at follow-up, mm (IQR) & $1(0-5)$ & $7.5(5-8)$ & 0.002 \\
\hline SPT fresh raw egg white at diagnosis, mm (IQR) & $14(12-20)$ & $13(12-15)$ & 0.80 \\
\hline SPT fresh raw egg white at follow-up, mm (IQR) & $5(2-9)$ & $12(10-13)$ & 0.048 \\
\hline \multicolumn{4}{|l|}{ Median ratio } \\
\hline sIgE egg white at follow-up/diagnosis, \% (IQR) & $16.0(16.6-38.0)$ & $20.7(8.6-50.0)$ & 0.533 \\
\hline \multicolumn{4}{|l|}{ Median ratio } \\
\hline sIgE ovomucoid at follow-up/diagnosis, \% (IQR) & $20.0(2.0-45.0)$ & $23.3(6.7-66.0)$ & 0.61 \\
\hline \multicolumn{4}{|l|}{ Median ratio } \\
\hline SPT egg white at follow-up/diagnosis, \% (IQR) & $14.0(0-116.0)$ & $112.0(83.0-150.0)$ & 0.075 \\
\hline \multicolumn{4}{|l|}{ Median ratio } \\
\hline SPT raw egg at follow-up/diagnosis, \% (IQR) & $42.0(0-74)$ & $94.0(80-100)$ & 0.012 \\
\hline
\end{tabular}

prevalence, SPT size to peanut, sIgE to peanut and Ara h 2 at diagnosis and severity of original reaction. SCORAD at the time of diagnosis was significantly higher in those lost to follow-up (Table E2*).

The median age of those followed up and challenged was 9 years and 6 months (114 (IQR 77 - 148) months). Six of 19 patients (31.6\%) had accidentally ingested peanut during the 5 years since the initial study, of whom 4 had reacted at the time of accidental ingestion, 1 with an anaphylactic reaction.

Three of 19 children (15.8\%) acquired tolerance at a median age of 8 years and 4 months (100 (IQR 68 - 157) months); 1/13 (7.7\%) children of mixed race outgrew their allergies at the age of 68 months; and 2/6 (33.3\%) of black Africans outgrew their peanut allergy at a median age of 128.5 (IQR 100 - 157) months (inter-ethnic difference in outgrowing peanut allergy, $p=0.16$ ).

Four of 19 patients followed up for peanut allergy had severe anaphylactic symptoms originally. Of 3 patients who outgrew their peanut allergy, none had severe symptoms originally, whereas $4 / 16$ (25\%) of those who did not outgrow their peanut allergy had previously experienced severe symptoms $(p=0.33)$.

\section{Allergic comorbidity}

Nine of 19 participants (47.4\%) had asthma at reassessment compared with $8 / 19$ (42.1\%) with asthma at the time of the original study; hence, there was a $5 \%$ increase in asthma over 5 years in this group. At diagnosis, of those who subsequently outgrew their peanut allergy, $1 / 3(33.3 \%)$ had asthma, whereas $7 / 16(43.8 \%)$ of those with persistent peanut allergy had asthma $(p=0.74)$. At the time of the follow-up study, of those who outgrew their peanut allergy, 2/3 (66.7\%) had asthma, and of those who did not outgrow their peanut allergy, $7 / 16(43.8 \%)$ had asthma $(p=0.46)$.

The median SCORAD at study entry in the group that subsequently outgrew their peanut allergy was 31 (IQR 22 - 46) compared with 41.5
(IQR 32 - 52) in those who did not outgrow their peanut allergy ( $p=0.22$ ). In the follow-up study, the median SCORAD in those who outgrew their peanut allergy (26.5, IQR 19 - 31) and in those who did not (26, IQR 20 - 45) $(p=0.72)$ had decreased, but was in the moderate range.

Specific IgE tests to peanut and Ara $\mathrm{h} 2$ at diagnosis and follow-up in relation to tolerance acquisition

The sIgE to peanut and Ara h 2 at diagnosis and follow-up are compared in patients who outgrew their peanut allergy and in those with persistent allergy (Table 2).

\section{Skin-prick tests to peanut at diagnosis and follow-up in relation to tolerance acquisition}

SPTs to peanut at diagnosis and follow-up are compared in those who outgrew their allergy and those with persistent allergy (Table 2).

\section{Ratios of follow-up results to original results}

The ratios of median sIgE peanut at follow-up compared with those at diagnosis are depicted in Table 2; ratios for Ara h 2 sIgE and SPTs to peanut at follow-up v. at diagnosis are also depicted in Table 2.

\section{Discussion}

This is the first SA study assessing factors influencing tolerance development in children with $\mathrm{AD}$ and concomitant food allergy in low- and middle-income settings. Egg and peanut allergies were predominant in the cohort, with large enough numbers for follow-up 5 years after the diagnosis of food allergy.

Seventy-two percent of children with egg allergy outgrew their allergy 5 years after diagnosis at a median age of 8 years and 4 months. This is similar to tolerance acquisition rates in other countries, ${ }^{[13]}$ but higher than that in several studies from tertiary centres. ${ }^{[1,15]}$ The rate of tolerance acquisition for egg did not differ according to ethnicity. 
Table 2. Factors affecting tolerance acquisition in peanut allergy

\begin{tabular}{|c|c|c|c|}
\hline Factor & $\begin{array}{l}\text { Children who } \\
\text { acquired tolerance to } \\
\text { peanut }\end{array}$ & $\begin{array}{l}\text { Children with persistent } \\
\text { peanut allergy }\end{array}$ & $\begin{array}{l}\text { Difference } \\
(p \text {-value })\end{array}$ \\
\hline Asthma at diagnosis, $\%$ & 33.3 & 43.8 & 0.74 \\
\hline Asthma at follow-up, \% & 66.7 & 43.8 & 0.46 \\
\hline Median SCORAD at diagnosis (IQR) & $31.0(22-46)$ & $41.5(32-52)$ & 0.22 \\
\hline Median SCORAD at follow-up (IQR) & $26.5(19-31)$ & $26(20-45)$ & 0.72 \\
\hline sIgE peanut at diagnosis, kU/L (IQR) & $12.7(1.8-23.4)$ & $17.2(10.7-87.8)$ & 0.31 \\
\hline sIgE peanut sIgE at follow-up, kU/L (IQR) & $0.22(0.1-0.3)$ & $31.9(8.9-56.9)$ & 0.02 \\
\hline sIgE Ara h 2 at diagnosis, kU/L (IQR) & $1.7(0.84-2.63)$ & $16.9(7.9-33.6)$ & 0.02 \\
\hline sIgE Ara h 2 at follow-up, kU/L (IQR) & $0.18(0.10-0.26)$ & $18.6(4.63-24.90)$ & 0.03 \\
\hline SPT peanut at diagnosis, mm (IQR) & $10(4-11)$ & $14(11.5-18.5)$ & 0.04 \\
\hline SPT peanut at follow-up, mm (IQR) & $5.5(5.0-8.0)$ & $12.3(9.2-13.2)$ & 0.02 \\
\hline \multicolumn{4}{|l|}{ Median ratio } \\
\hline sIgE peanut at follow-up/diagnosis, \% (IQR) & $4.0(3-5)$ & $99.5(57-162)$ & 0.02 \\
\hline \multicolumn{4}{|l|}{ Median ratio } \\
\hline sIgE Ara h 2 at follow-up/diagnosis, \% (IQR) & $8.0(6-10)$ & $71.5(39-137)$ & 0.03 \\
\hline \multicolumn{4}{|l|}{ Median ratio } \\
\hline SPT peanut at follow-up/diagnosis, \% (IQR) & $73.0(55-120)$ & $75.0(61-100)$ & 0.86 \\
\hline
\end{tabular}

Asthma prevalence was high in participants with egg allergy and increased over the follow-up period, both in those who outgrew their allergy and in those with persistent allergy. This is in keeping with previous studies showing egg allergy as a significant risk factor for asthma development. ${ }^{[28]} \mathrm{AD}$, although reducing somewhat in severity over time, remained of moderate severity in the majority of participants -in those who had acquired tolerance to egg and in those with persistent allergy.

The median sIgE to both egg white and ovomucoid was significantly higher at diagnosis and follow-up in those with persistent egg allergy than in those who developed tolerance. At diagnosis, sIgE egg white and ovomucoid $>100 \mathrm{kU} / \mathrm{L}$ were associated with persistent allergy after 5 years; and sIgE egg white $\leq 9.0 \mathrm{kU} / \mathrm{L}$ and ovomucoid $\leq 2.0 \mathrm{kU} / \mathrm{L}$ at diagnosis were associated with tolerance development. At followup, sIgE egg white $\geq 20.0 \mathrm{kU} / \mathrm{L}$ and ovomucoid $\geq 23.0 \mathrm{kU} / \mathrm{L}$ were associated with persistent allergy, while sIgE egg white $\leq 0.70 \mathrm{kU} / \mathrm{L}$ and ovomucoid $\leq 0.16 \mathrm{kU} / \mathrm{L}$ were associated with tolerance.

However, the trend in those who outgrew their egg allergy was a reduction in sIgE to egg and ovomucoid over time, suggesting that more children might outgrow their egg allergy.

The size of the original SPT to both egg extract and raw egg did not significantly influence the chances of developing tolerance. However, the SPT area to egg extract and fresh raw egg white at follow-up was significantly larger in the group with persistent egg allergy. At followup, an SPT to egg-white extract $\geq 7.5 \mathrm{~mm}$ and fresh egg $\geq 12 \mathrm{~mm}$ was associated with persistence, while an SPT to egg-white extract $\leq 1 \mathrm{~mm}$ and raw egg $\leq 5 \mathrm{~mm}$ was associated with tolerance. The SPT to fresh raw egg was generally higher than that of the egg extract and in many cases remained positive (median $5 \mathrm{~mm}$ ) even if the child had outgrown the egg allergy. Therefore, the clinician should strongly consider an egg challenge if the egg extract result becomes negative, even in the presence of a positive raw egg SPT.

In this cohort, $15.8 \%$ of children outgrew their peanut allergy after 5 years. This is in keeping with previous studies showing acquisition of tolerance in $\sim 20 \%$ of peanut-allergic children. ${ }^{[20-24]}$ Asthma prevalence remained stable in those who retained and in those who outgrew their peanut allergy. Similar to the group with egg allergy, eczema severity reduced over time, but remained of moderate severity in the majority of participants.

The median sIgE to peanut at diagnosis was not significantly higher in those who persisted in their allergy; however, it was significantly higher for Ara h 2 in the persistence group. At diagnosis, sIgE to Ara h $2 \geq 16.95 \mathrm{kU} / \mathrm{L}$ was associated with persistence, and to Ara h $2 \leq 1.7 \mathrm{kU} / \mathrm{L}$ with tolerance development. At follow-up, sIgE to peanut $\geq 31.9 \mathrm{kU} / \mathrm{L}$ and Ara $\mathrm{h} 2 \geq 18.6 \mathrm{kU} / \mathrm{L}$ was associated with persistent allergy, while sIgE to peanut $\leq 0.22 \mathrm{kU} / \mathrm{L}$ and Ara $\mathrm{h} 2$ $\leq 0.18 \mathrm{kU} / \mathrm{L}$ was associated with tolerance. At diagnosis, an SPT $\geq 14 \mathrm{~mm}$ was associated with persistence and an SPT $\leq 10 \mathrm{~mm}$ with tolerance acquisition, while at follow-up, an $\mathrm{SPT} \geq 12 \mathrm{~mm}$ was associated with persistence and an SPT $\leq 5.5 \mathrm{~mm}$ with tolerance acquisition. The ratio of the follow-up peanut IgE compared with the original IgE was significantly lower in the group who outgrew their peanut allergy, indicating a significant fall in IgE over time.

In peanut allergy, neither ethnicity nor the number of allergies at diagnosis affected the development of tolerance.

This study, in a low- and middle-income socioeconomic setting in an African country, mirrors the substantial differences in the natural history of egg and peanut allergy demonstrated in previous studies in higher socioeconomic settings, indicating that differences in the immunology of egg and peanut allergy seem ubiquitous across different geographical, socioeconomic and ethnic settings. A greater understanding of the reasons for such differences may help to provide an opportunity for clinical or immunological interventions in food allergy. Understanding the natural history of common food allergens in the SA setting allows for appropriate counselling of foodallergic children, and provides guidance to the treating clinicians on avoidance and reintroduction of foods as nutritious, cost-effective and ubiquitous as egg and peanut in a resource-constrained setting.

\section{Study strengths and limitations}

Strengths of the study are that every patient underwent a food challenge irrespective of SPTs and SIgE levels. The small cohort size and $20-30 \%$ loss-to-follow-up rate may have affected the conclusions. Maximal effort was made to phone, email, send letters and do home 
visits to follow up patients, but the migrant nature of this population meant that many had moved to other areas of the country.

\section{Conclusions}

In $\mathrm{SA}$ children with $\mathrm{AD}$, egg allergy was outgrown in $72.2 \%$ and peanut allergy in $15.8 \% 5$ years after diagnosis. This is in keeping with figures derived from studies in higher socioeconomic settings, and can help to guide counselling of patients with allergies to these foods of high nutritional value. Allergic comorbidity remained high, with asthma increasing over time and $\mathrm{AD}$ remaining moderate in severity in the cohort overall. Low sIgEs to peanut, Ara h 2, egg white and ovomucoid at diagnosis and follow-up may indicate possible development of tolerance and guide the appropriate timing of food challenges. SPTs at follow-up (egg and peanut) and at diagnosis (peanut allergy only) can also be used to help to prognosticate allergy persistence and guide the appropriate timing of challenges.

*Tables E1 and E2 are available from the corresponding author on request. Declaration. None.

Acknowledgements. We thank Sister Lucia Volkwyn for assistance with the food challenges.

Author contributions. CLG: designed and executed the study and wrote up the article; MEL: reviewed the article; GdT: contributed to the study design and reviewed the article.

Funding. The study was partly funded by a research award to CLG in 2014/2015 from the Allergy Society of South Africa.

Conflicts of interest. None.

1. Hu Y, Chen J, Li H. Comparison of food allergy prevalence among Chinese infants in Chongqing, 2009 versus 1999. Pediatr Int 2010;52(5):820-882. https://doi.org/10.1111/j.1442-200X.2010.03166.X 2. Sampson HA. Update on food allergy. J Allergy Clin Immunol 2004;113(5):805-819. https://doi. org/10.1016/S0091-6749(04)01145-5

3. Rance F, Grandmottet X, Grandjean H. Prevalence and main characteristics of schoolchildren diagnosed with food allergies in France. Clin Exp Allergy 2005;35(2):167-172. https://doi.org/10.1111/ jiagnosed with food aller

4. Pereira B, Venter C, Grundy J, et al. Prevalence of sensitization to food allergens, reported adverse reaction to foods, food avoidance, and food hypersensitivity among teenagers. J Allergy Clin Immunol 2005;116(4):884-892. https://doi.org/10.1016/j.jaci.2005.05.04

5. Osterballe M, Hansen TK, Mortz CG, et al. The prevalence of food hypersensitivity in an unselected population of children and adults. Pediatr Allergy Immunol 2005;16(7):567-573. https://doi.org/10.1111/j.1399-3038.2005.00251.x

6. Osborne NJ, Koplin JJ, Martin PE, et al. Prevalence of challenge-proven IgE-mediated food allergy Immunol 2011;127(3):668-676. https://doi.org/10.1016/j.jaci.2011.01.039
7. Burney P, Summers C, Chinn S, et al. Prevalence and distribution of sensitization to foods in the European Community Respiratory Health Survey: A EuroPrevall analysis. Allergy 2010;65(9):1182European Community Respiratory Health Survey: A EuroP

8. Dalal I, Binson I, Reifen R, et al. Food allergy is a matter of geography after all: Sesame as a major cause of severe IgE-mediated food allergic reactions among infants and young children in Israel. Allergy 2002;57(4):362-365. https://doi.org/10.1034/j.1398-9995.2002.1s3412.x

9. Marklund B, Ahlstedt S, Nordstrom G, et al. Health-related quality of life among adolescent with allergy-like conditions - with emphasis on food hypersensitivity. Health Qual Life Outcomes 2004;2:65. https://doi.org/10.1186/1477-7525-2-65

10. Eigenmann PA, Sicherer SH, Borkowski TA, et al. Prevalence of IgE-mediated food allergy among children with atopic dermatitis. Pediatrics 1998:101(3):e8. https://doi.org/10.1542/peds.101. 3 e8

1. Figenmann $\mathrm{PA}, \mathrm{C}$ ana A-M. Dingnosis of IgE-mediated food allergy among Swiss children with Eigenmann PA, Calza A-M. Diagnosis of IgE-mediated food allergy among Swiss children with atopic dermatitis. Pediatr Allergy Immunol 2000;11(2):95-100. https://doi.org/10.1034/j.1399-
$3038.2000 .00071 . x$ $3038.2000 .00071 . x$

2. Gray CL, Levin ME, du Toit G. Food allergy in South African children with atopic dermatitis. Pediatr Allergy Immunol 2014;25(6):572-579. https://doi.org/10.1111/pai.12270

13. Boyano-Martinez T, Garcia-Ara C, Diaz-Pena JM, Martin-Esteban M. Prediction of tolerance on the basis of quantification of egg white specific IgE antibodies in children with egg allergy. J Allergy Clin Immunol 2002;110(2):304-309. https://doi.org/10.1067/mai.2002.126081

14. Savage JH, Matsui EC, Skripak JM, Wood RA. The natural history of egg allergy. J Allergy Clin Immunol 2007;120(6):1413-1418. https://doi.org/10.1016/j.jaci.2007.09.040

15. Taniguchi H, Ogura K, Sato S, et al. Natural history of hen's egg allergy from 6 - 12 years of age J Allergy Clin Immunol 2017;139(2):AB137. https://doi.org/10.1016/j.jaci.2016.12.451

16. Ford RP, Taylor B. Natural history of egg hypersensitivity. Arch Dis Child 1982:57(9):649-652. https://doi.org/10.1136/adc.57.9.649

17. Dannaeus A, Inganas M. A follow-up study of children with food allergy: Clinical course in relation to serum IgE- and IgG-antibody levels to milk, egg and fish. Clin Allergy 1981;11(6):533-539. https://doi.org/10.1111/j.1365-2222.1981.tb02171.x

18. Kim JD, Kim SY, Sol IS, et al. Influential factors in tolerance acquisition of IgE-mediated egg allergy in children. J Allergy Clin Immunol 2017;139(2):AB144. https://doi.org/10.1016/j.jaci.2016.12.474

19. Jarvinen K-M, Beyer K, Vila L, et al. Specificity of IgE antibodies to sequential epitopes of hen's egg ovomucoid as a marker for persistence of egg allergy. Allergy 2007;62(7):758-765. https://doi. org/10.1111/.1398-9995.2007.01332.x

20. Hourihane JO'B, Roberts SA, Warner JO. Resolution of peanut allergy: Case control study. BMJ 1998:316(7140):1271-1275. https://doi.org/10.1067/mpd.2000.109376

21. Van der Leek TK, Liu AH, Stefanski K, Blacker B, Bock SA. The natural history of peanut allergy in young children and its association with serum peanut-specific IgE. J Pediatr 2000;137(6):749-755. https://doi.org/10.1067/mpd.2000.109376

22. Spergel JM, Beausoleil JL, Pawlowski NA. Resolution of childhood peanut allergy. Ann Allergy Asthma Immunol 2000;85(6):473-476. https://doi.org/10.1016/s1081-1206(10)62574-4

23. Peters RL, Koplin JJ, et al. Natural history of peanut allergy and predictors of resolution in the first 4 years of life: A population-based assessment. J Allergy Clin Immunol 2015;135(5):1257-1266. https://doi.org/10.1016/j.jaci.2015.01.002

24. Fleischer DM, Conover-Walker MK, Christie L, Burks W, Wood R. The natural progression of peanut allergy: Resolution and the possibility of recurrence. J Allergy Clin Immunol 2003;112(1):183-189. https://doi.org/10.1067/mai.2003.1517

25. Skolnick HS, Conover-Walker MK, Koerner CB, Sampson HA, Burks W, Wood RA. The natural history of peanut allergy. J Allergy Clin Immunol 2001;107(2):367-374. https://doi.org/10.1067/ mai.2001.112129

26. Gray CL, Levin ME, du Toit G. Egg sensitization, allergy and component patterns in African children with atopic dermatitis. Pediatr Allergy Immunol 2016;27(7):709-715. https://doi org/10.1111/pai.12615

27. Gray CL, Levin ME, du Toit G. Ethnic differences in peanut allergy patterns in South African children with atopic dermatitis. Pediatr Allergy Immunol 2015;26(8):721-730. https://doi. org/10.1111/pai.12459

28. Tariq SM, Matthews SM, Hakim EA, Arshad SH. Egg allergy in infancy predicts respiratory allergy disease by 4 years of age. Pediatr Allergy Immunol 2000;11(3):162-167. https://doi.org/10.1034 j.1399-3038.2000.00077.x

Accepted 9 October 2018 